\title{
Co-Evolutionary Multi-Objective Multidisciplinary Design Optimization for Hypersonic Vehicles
}

\author{
D. Zhang \\ College of Astronautics \\ Northwestern Polytechnical University \\ Xi' an, Shannxi, P.R. China
}

\author{
S. Tang \\ Notional Key Laboratory of Aerospace Flight Dynamics \\ Northwestern Polytechnical University, \\ Xi'an, Shannxi, P.R. China
}

\begin{abstract}
Integrated design of airframe/propulsion of hypersonic vehicles is a problem of multi-objective multidisciplinary design optimization (MDO) for complex coupled systems in nature. In order to implement a MDO on airframe/propulsion integration, firstly the design structure matrix was established based on the analysis of coupling relationships among disciplines for airframe/propulsion integration; secondly, the system optimization model and disciplinary optimization models for airframe/propulsion integration were proposed; finally, simulations for the multiobjective multidisciplinary design optimization of airframe/propulsion integration of hypersonic vehicles were conducted by means of co-evolutionary method and a satisfactory Pareto optimal solution set was obtained.
\end{abstract}

Keywords-airframe; propulsion; hypersonic vehicle; multidisciplinary; $M D O$

\section{INTRODUCTION}

For the airframe/propulsion integration of hypersonic vehicles, strong couplings exist among disciplines such as structural mechanics, aerothermodynamics, combustion, trajectory and control, and geometric profile in addition to the coupling between aerodynamics and propulsion. Integrated design of airframe/propulsion for hypersonic vehicles is a typical multi-objective multidisciplinary design optimization problem due to its intrinsic characteristic of multidisciplinary coupling.

The purpose of multi-objective multidisciplinary design optimization of airframe/propulsion integration is to find the set of Pareto optimal solutions that satisfy constraints and requirements on performance of airframe/propulsion integration, and furthermore to provide several design plans that are optional for designers, which is of great importance for the integrated design of airframe/propulsion for hypersonic vehicles.

MDO is a methodology to design complex systems and subsystems by exploring and making full use of synergic mechanisms of interactions in systems [1]. Details about the developments of the main ideology, contents and key techniques of MDO are elaborated in papers [2-4].

\section{Multi-OBJective MultidisciplinARy DESIGN OPTIMIZATION OF AIRFRAME/PROPULSION INTEGRATION}

Based on the analysis of coupling relationships between disciplines, the strongly coupled system of a hypersonic vehicle is decomposed into multidisciplinary systems that are relatively independent and autonomous by means of appending design variables. In the meantime, the consistency of interdisciplinary coupling constraints is enforced by applying equation constraints in each discipline. In such a way, all disciplines and co-evolutionary multi-objective optimization algorithm can be combined to establish the mappings between populations and disciplines, so that a multi-population self-adaptive co-evolutionary design optimization model is generated. The Pareto solution set is obtained through the storage mechanism of Pareto solutions.

\section{A. Design Optimization Model}

System performance indicators, design variables and constraints play fatal roles for multi-objective multidisciplinary design optimization of airframe/propulsion integration of hypersonic vehicles. For a hypersonic vehicle, the airframe is the part that generates lift for the vehicle and the quality of its profile directly affects the aerodynamic performance of the vehicle and thereafter the accomplishment of flight missions. As a result, the L/D ratio of the airframe is an important performance indicator, which is often used to measure the aerodynamic performance of the vehicle. The performance of propulsion system is usually indicated by thrust coefficient Ct. Heat flux q or temperature $\mathrm{Tb}$ along the central line on outer surface of airframe is taken as the indicator of aero thermo dynamic characteristic.

Constraints on systems in a hypersonic vehicle includes constraint on inlet starting, constraint on boundary layer separation, combustion condition of combustor and constraint on forebody length, etc. Constraint on inlet starting means Kantrowitz condition [5]:

$$
\frac{A_{2}}{A_{1}} \geq\left[\frac{\gamma-1}{\gamma+1}+\frac{2}{(\gamma+1) M_{1}^{2}}\right]^{\frac{1}{2}}\left[\frac{2 \gamma}{\gamma+1}-\frac{\gamma-1}{(\gamma+1) M_{1}^{2}}\right]^{\frac{1}{\gamma-1}}
$$

Where, ${ }^{A_{2}}$ is the area of inlet throat, ${ }^{A_{1}}$ is incoming flow capture area, ${ }^{M_{1}}$ is Mach number behind the shock induced by the outer compression surface.

With respect to the disadvantage of Kantrowitz condition which is too conservative, it is proposed by paper [6] that inlet starting is determined by the following empirical formula:

$$
\frac{H_{2}}{H_{1}} \geq-3.25+2.17 M a_{\text {cruise }}-0.017 M a_{\text {cruise }}^{2}
$$


Constraint on boundary layer separation [6] means that the ratio of Mach numbers ahead of and behind shock satisfies a certain condition.

For a laminar boundary layer

$$
\left(\frac{M_{a}}{M_{f}}\right)_{l a \min a r}<0.898(3)
$$

For a turbulent boundary layer

$$
\left(\frac{M_{a}}{M_{f}}\right)_{\text {turbulent }}<0.762(4)
$$

Where, ${ }_{a}$ is Mach number behind the shock, $M_{f}$ is the Mach number ahead of the shock.

Combustion condition is that in order to guarantee the normal combustion in the combustor, properties of the air flow entering the combustor are constrained [7]:

$$
\begin{gathered}
0.5 \mathrm{~atm}<P_{\text {com, in }}<10 \mathrm{~atm}(5) \\
2.0<M a_{\text {com, in }}<3.0(6)
\end{gathered}
$$

By applying the constraint on forebody length, the length of the forebody is constrained in order to satisfy the requirements of internal volume and engine structure:

$$
L_{O C} \leq 2.7 \quad(7)
$$

Based on the above analysis, the multi-objective multidisciplinary design optimization of airframe/propulsion of hypersonic vehicles can be described by a system-level optimization model and three discipline-level optimization models.

The system-level optimization model:

$$
\begin{aligned}
& \text { Maximize }\left\{\frac{C_{L}}{C_{D}}, C_{T}, \frac{1}{T_{b}}\right\} \\
& \text { s.t. } \quad X_{m \text { in }} \leq X \leq X_{\max }
\end{aligned}
$$

Discipline-level optimization models include models of disciplines such as aerodynamics, propulsion and aerothermodynamics.

Discipline of aerodynamics:

$$
\begin{aligned}
& \text { Maximize } \frac{C_{L}}{C_{D}} \\
& \text { s.t. } \quad x_{1 \min } \leq x_{1} \leq x_{1 \max }, \quad L_{O C} \leq 2.7
\end{aligned}
$$

Discipline of propulsion:

$$
\begin{array}{ll}
\text { Maximize } \quad C_{T} \\
\text { s.t. } & \delta_{1}+\delta_{2}+\delta_{3}=\delta_{4}+\delta_{5}, \quad M_{a 3} \leq 5.0(10) \\
& x_{2 \min } \leq x_{2} \leq x_{2 \max }, \quad \theta_{3} \leq \theta_{2} \leq \theta_{1}
\end{array}
$$

In the meantime, the discipline-level model of propulsion should satisfy constraint on inlet starting, constraint on boundary layer separation and combustion condition of combustor.

Discipline of aerothermodynamics:

$$
\begin{aligned}
& \text { Maximize } \frac{1}{T_{b}} \\
& \text { s.t. } \quad x_{3 \min } \leq x_{3} \leq x_{3 \max }
\end{aligned}
$$

It is impossible to set all geometric parameters that describe the vehicle profile to be design variables. Under the condition that geometric parameters on the transverse direction of the vehicle, i.e. lateral edge parameters, do not change, the geometric parameters that characterize the shapes of upper and lower surfaces of the vehicle are chosen to be design optimization variables. These 12 variables include angles of deflection $\delta_{i}(i=1,2,3,4)$ that characterize the geometry of the lower surface of the forebody, angles $\theta_{j}(j=1,2,3)$ that characterize the geometry of the rear nozzle, the radius of curvature of the vehicle nose $R_{n}$ and the geometric parameters $Y_{O H}, Y_{O G}$ and $L_{O H}, L_{O G}$ that characterize the geometry of the upper surface of the vehicle.

\section{B. Co-Evolutionary Multi-Objective Multidisciplinary Design Optimization (Cmomdo) Algorithm}

Through the analysis of coupling relationships among disciplines involved in airframe/propulsion integration of hypersonic vehicles, the multi-objective multidisciplinary design optimization of airframe/propulsion integration can be formulated as a 2-level MDO structure, based on which a system-level optimization model and models about each disciplines were established. After that, co-evolutionary multi-objective multidisciplinary design optimization (CMOMDO) algorithm was employed to implement the multi-objective multidisciplinary design optimization of airframe/propulsion integration.

On the foundation of co-evolution of populations and their neighborhoods, CMOMDO algorithm is a highly efficient random search algorithm which combines local search of partial solutions and global search of complete solutions. Based on this ideology, a CMOMDO code for airframe/propulsion integration of hypersonic vehicles was developed on the platform of Matlab 7.0. The flow chart is shown in Figure. 1. 


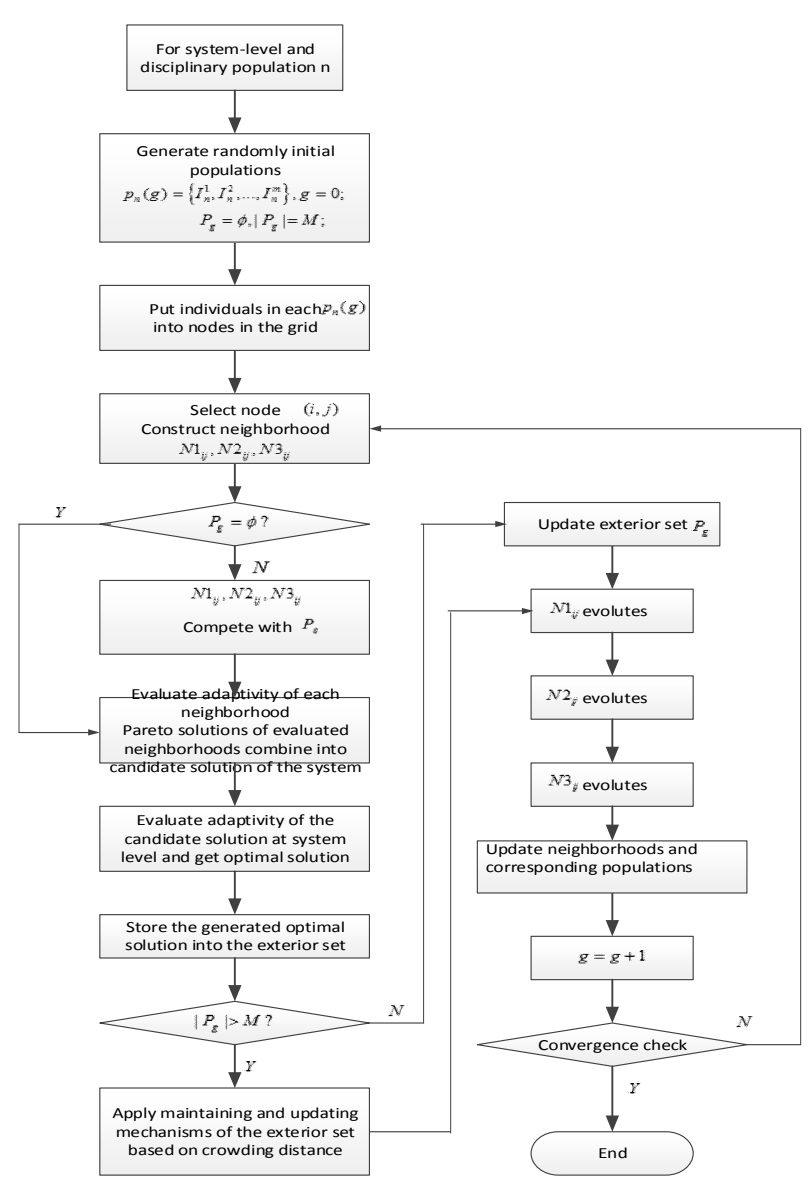

FIGURE I. FLOW CHART OF CMOMDO FOR AIRFRAME/PROPULSION INTEGRATION OF HYPERSONIC VEHICLES.

\section{Simulation RESUlTS AND ANALYSIS}

Through simulations, the Pareto optimal solution set and Pareto frontier for the multi-objective multidisciplinary design optimization of airframe/propulsion integration of hypersonic vehicles were obtained. Figure. 2 demonstrates the Pareto frontier obtained by CMOMDO algorithm for airframe/propulsion integration. Table 1 shows the comparison between performances of configurations on the boundary of Pareto frontier and the performance of the base configuration.

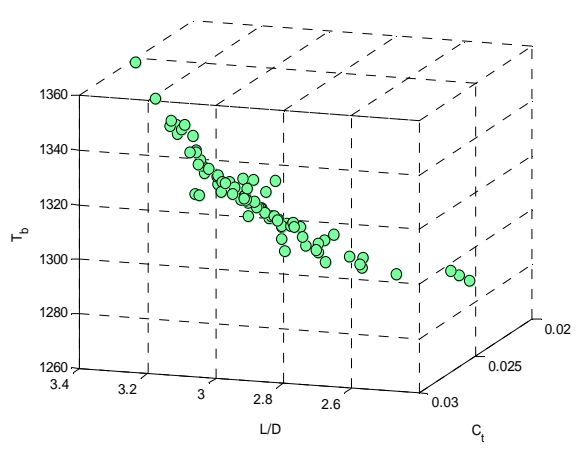

FIGURE II. PARETO FRONTIER OBTAINED BY CMOMDO ALGORITHM FOR AIRFRAME/PROPULSION INTEGRATION.
TABLE I. COMPARISON BETWEEN PERFORMANCES OF PARETO FRONTIER AND BASE CONFIGURATION.

\begin{tabular}{|c|c|c|c|c|}
\hline \multirow{2}{*}{ Parameters } & \multirow{2}{*}{$\begin{array}{l}\text { Baseline } \\
\text { configuration }\end{array}$} & \multicolumn{3}{|c|}{ Pareto Boundary layer solution } \\
\hline & & $\operatorname{Max} L / D$ & $\operatorname{Max} C_{t}$ & $\operatorname{Min} T_{b}$ \\
\hline$\delta_{1}$ & $0.636^{0}$ & 5.38740 & 4.69450 & 3.64720 \\
\hline$\delta_{2}$ & $1.728^{\circ}$ & 1.70220 & 1.35430 & 2.96610 \\
\hline$\delta_{3}$ & 5.6340 & 2.54340 & 3.61170 & 1.66620 \\
\hline$\delta_{4}$ & 2.5280 & 7.75330 & 8.64430 & 5.94980 \\
\hline$\theta_{1}$ & 15.000 & 8.29850 & 21.97170 & 10.15240 \\
\hline$\theta_{2}$ & 8.000 & 7.39210 & 14.76620 & 7.70490 \\
\hline$\theta_{3}$ & 0.000 & 5.59080 & 12.7799 & 6.65280 \\
\hline$R_{n}$ & 0.01 & 0.012 & 0.0319 & 0.04510 \\
\hline$L_{O H}$ & 2.00 & 2.1871 & 2.1899 & 1.9036 \\
\hline$Y_{O H}$ & 0.0785 & 0.0591 & 0.1245 & 0.0937 \\
\hline$L_{O G}$ & 4.300 & 4.2565 & 4.1286 & 4.1936 \\
\hline$Y_{O G}$ & 0.140 & 0.1812 & 0.1245 & 0.1389 \\
\hline
\end{tabular}

Conclusions are drawn through analysis of Table 1:

1) Aerodynamic characteristic of hypersonic vehicles with airframe/propulsion depends mainly on the deflection angle of air flow $\delta$ of forebody and the radius of curvature at the vehicle nose $R_{n}$. Both the increase of deflection angle and the reduction of radius of curvature can increase the lift for the vehicle body. In the meantime, drag on forebody is also increased due to the increase of deflection angle of air flow and larger radius of curvature at the vehicle nose leads to greater drag on the upper surface of the vehicle nose, which reduces the lift-to-drag characteristic of the vehicle. This is the reason why drag increase faster than lift.

2) The aero thermo dynamic performance of the vehicle mainly depends on the radius of curvature at nose. If the radius of curvature increases, heat flux at the vehicle nose is relatively small, which is indicated by relatively low temperature at the vehicle nose. If the radius of curvature decreases, heat flux at the vehicle nose is relatively large, which is indicated by relatively high temperature at the vehicle nose.

3) The propulsion performance of the vehicle is mainly dependent on the angle of rear nozzle $\theta$. The more completely the rear nozzle expands, the greater the Mach number after the air flow accelerates is. In the meantime, the thrust also depends on compression in the forebody. The greater the deflection angle of air flow is, the more heavily air flow is compressed, the less the energy loss due to compression is, the larger the thrust is.

\section{CONCLUSIONS}

In the work reported by this paper, the co-evolutionary multi-objective multidisciplinary design optimization for airframe/propulsion integration of hypersonic vehicles was investigated. The system-level and discipline-level optimization models for airframe/propulsion integration of hypersonic vehicles were established. Knowledge of each discipline and the CMOMDO algorithm were merged to 
generate a model for the multi-population self-adaptive coevolutionary design optimization process. The Pareto optimal solution set that satisfies requirements of performance and constraints was obtained by means of simulations and analysis of cases for the co-evolutionary multi-objective multidisciplinary design optimization for airframe/propulsion integration of hypersonic vehicles.

\section{ACKNOWLEDGEMENT}

This study was supported by the Fundamental Research Funds for the Central Universities (NO. 3102014KYJD008).

\section{REFERENCES}

[1] Giesing J P, Barthelemy J M. A summary of industry MDO applications and needs. An AIAA White Paper, 1998.

[2] WANG Shu-he, HE Lin-shu. The summarization of multidisciplinary design optimization for flight vehicles[J]. Journal of Astronautics, 2004, 25(6).(In chinese).

[3] JIA Jian-dong, YAO Wei-xing, WU De-hai. An Introduction to Multidisciplinary Design of Aircraft Optimization Technique[J]. Aerospace Science and Technology, 2005,(6):21-25. (In chinese).

[4] Kevin G B, Geojoe K, Thomas A G. Advancements in multidisciplinary design optimization applied to hypersonic vehicles to achieve closure[R]. AIAA 2008-2591.

[5] Thomas J B. CFD Modeling of the Hypersonic Inlet Staring Problem [R]. AIAA-2006-123, 2006.

[6] Billig F S. Research on Supersonic Combustion [J]. Journal of Propulsion and Power, 1993, 9(4)499-514.

[7] W H Heiser, David T P. Hypersonic airbreathing propulsion, AIAA Education Serises, ISBN:1-56347-035-7, Published by American Institute of Aeronaustics and Astronaustics, 1994. 